\title{
KORRELATIONEN ZWISCHEN MERKMALHAFTEN ODER MERKMALLOSEN SYNTAKTISCHEN VARIANTEN UND VERSCHIEDENEN GRADEN DER TEXTSORTENKOMPLEXITÄT
}

\section{GRUNDANNAHMEN}

Gemäß der Grundannahme über das Verhalten von sprachlichen Varianten gilt folgendes (vgl. Orešnik, Snedec, Teržan, Trobevšek-Drobnak 1991: 5-6):

(1) Verstärkte Konstruktionen bzw. starke Varianten (d.h. semantisch und/oder funktional komplexere als auch formal komplexere Formen, rezipientenfreundlicher) behaupten sich zumindest im Variantenstadium vorzugsweise in komplexerer grammatischer Umgebung.

(2) Geschwächte Konstruktionen bzw. schwache Varianten (d.h. semantisch allgemeinere und/oder funktional undurchsichtigere als auch formal einfachere Formen, produzentenfreundlicher) behaupten sich zumindest im Variantenstadium vorzugsweise in weniger komplexer grammatischer Umgebung.

(3) Die Komplexität der grammatischen Umgebung wird hauptsächlich gemäß den Annahmen in der Natürlichkeitstheorie über die Markiertheit bzw. Natürlichkeit sprachlicher Kategorien und Elemente beurteilt (vgl. Mayerthaler 1981, Wurzel 1984, Dressler 1989 und Dotter 1990).

Mit "grammatischer Umgebung" ist in (1) und (2) primär der unmittelbare Kontext einer sprachlichen Konstruktion gemeint, d.h. die Phrase oder der Satz, in dem eine sprachliche Variante auftritt, sekundär aber auch Phänomene auf Übersatzsebene. Im vorliegenden Aufsatz soll die Annahme geprüft werden, ob zwischen verstärkten bzw. geschwächten Konstruktionen einerseits und bestimmten Textsorten andererseits ("Umgebung" einer sprachlichen Konstruktion im weitesten Sinne), eine Affinität besteht. Oder in verschärfter Form ausgedrückt:

(4) Verstärkte Konstruktionen bzw. starke Varianten treten im Variantenstadium bevorzugt in markierteren Grundtextsorten auf

(5) Geschwächte Konstruktionen bzw. schwache Varianten kommen im Variantenstadium bevorzugt in weniger markierten Grundtextsorten vor. 
Zur Überprüfung dieser These muß zunächst die Frage geklärt werden, welche Textsorten man als grundlegender und welche Textsorten man als abgeleitet oder spezieller betrachten kann. Nach Diewald (1991) lassen sich aufgrund dreier situativer Merkmale, die laut Diewald den höchsten Rang in der Hierarchie der textsortenkonstituierenden Merkmale einnehmen, unter den acht theoretisch möglichen Textsorten die folgenden Grundtextsorten (nach Diewald 1991: 296, terminologisch modifiziert von T.P. $)^{1}$ unterscheiden:

\begin{tabular}{|l|c|c|c|l|c|}
\hline (6) Grundtextsorten in Diewald (1991) nach situativen Faktoren geordnet \\
\hline Grundtextsorte (GTS) & {$[+\mathrm{d}]$} & {$[+\mathrm{m}]$} & {$[+\mathrm{f}]$} & Diewald 1991 & GTS \\
\hline mündlicher Nahdialog & + & + & + & Dialog & 1 \\
\hline mündlicher Ferndialog & + & + & - & Telefongespräch & 2 \\
\hline schriftlicher Ferndialog & + & - & - & Brief & 3 \\
\hline mündlicher Nahmonolog & - & + & + & Mündlicher Monolog & 4 \\
\hline schriftlicher Fernmonolog & - & - & - & Schriftlicher Monolog & 5 \\
\hline
\end{tabular}

Ich nehme an, daß zwischen den situativen Merkmalen die Korrelationen unter (7) angenommen werden können. Unter (7a) wird behauptet, (aa) daß Fernkommunikation vorzugsweise nicht-mündlich und nicht-dialogisch sowie (ab) daß nicht-mündliche Kommunikation vorzugsweise nicht-dialogisch betrieben wird, unter (7b) wird hingegen behauptet, daß (ba) Nahkommunikation vorzugsweise mündlich und dialogisch sowie (bb) daß mündliche Kommunikation vorzugsweise dialogisch abgwickelt wird.

(7a) $[-d]-->[-m]-->[-f]$

(7b) $[+\mathrm{d}]-->[+\mathrm{m}]--\rightarrow[+\mathrm{f}]$

Es wird außerdem angenommen, daß die Korrelation zwischen den Merkmalen unter (7a) stärker ist als die unter (7b). Diese Annahme wird aufgestellt aufgrund der Voraussetzung, daß der Dialog die weniger markierte Kommunikationsform und somit auch allgemeiner (d.h. in verschiedeneren Kommunikationssituationen) einsetzbar ist.

Zwei Grundtextsorten bilden aufgrund der verwendeten situativen Merkmale die beiden Grundtextsortenpole. Das sind der mündliche Nahdialog (GTS 1) als weniger markierter GTS-Pol und der schriftliche Ferndialog (GTS 5) als markierterer GTS-Pol. Die Markiertheit der übrigen Grundtextsorten ist zwischen diesen beiden Polen einzuordnen. Die Markiertheitsskala der Grundtextsorten (auf der Grundlage situativer Merkmale) läßt sich im Diagramm folgendermaßen darstellen (8):

1 Merkmalsachse SITUATION:

Kommunikationsrichtung [D]: [+d] [-d] [+dialogisch, -dialogisch]

Medium

[M]: $[+\mathrm{m}][-\mathrm{m}]$ [+mündlich, -mündlich]

Kontaktart

$[\mathrm{K}]:[+\mathrm{f}] \quad[-\mathrm{f}] \quad[$ +face-to-face, -face-to-face $]$ 


\begin{tabular}{ccccc} 
& & & [markierter] \\
\hline 1 & 2 & 3 & 4 & 5
\end{tabular}

\section{AFFINITÄTEN MERMALHAFTER/-LOSER FORMEN ZU BESTIMMTEN TEXTSORTEN}

\subsection{Variantenpaar 1: Freie Nominalisierung auf <-ung> mit Satzinhalt vs. Nebensatz}

Eine Nominalisierung in freier Fügung (9a) ist im Vergleich zu bedeutungsäquivalenten Nebensätzen (9b) eine syntaktisch geschwächte Konstruktion (vgl. Petrič 1990: 25-31, 1995c: 211-212). Nominalisierungen drücken bestimmte kommunikativ relevante Informationen nicht so explizit aus wie die entsprechenden Nebensätze und sind daher in ihrer Bedeutung weniger eindeutig. Im Gegensatz zu Nebensätzen sind Nominalisierungen verdichtende Ausdrucksformen für Satzinhalte, sie setzen beim Hörer bestimmte Inhalte als bekannt voraus (anaphorische Funktion) und verlangen daher von ihm mehr Inferenzarbeit. Aufgrund ihres geringeren formalen Aufwandes erleichtern Nominalisierungen (insbesondere prototypische Nominalisierungen, d.h. solche ohne attributive Ergänzungen) dem Sprecher die Textproduktion. Leicht erschließbare, voraussetzbare oder lokal weniger relevante Informationen können vom Textproduzenten ausgespart werden. Die Simplifizierung einer syntaktischen Struktur (sprecherseitiger Vorteil) wird allerdings mit Verdichtung der Informationsstruktur eines Satzteiles erkauft (hörerseitiger Nachteil). Eine lokal optimale syntaktische Simplifizierung wie die Linearisierung des komplexen Satzgefüges in (9b) schafft vermutlich eher eine sowohl produzenten- als auch rezipientenfreundlichere Äußerungsstruktur (9a), d.h. einen Kompromiß zwischen sprecherseitigem und hörerseitigem Aufwand.

(9a) erst einmal möchte ich sagen,+ daß ich bedaure,,++ daß ich die einzige der Frauen hier bin + ,

,+ die für die Abschaffung des Paragraphen eintreten,+ . (Heutiges Deutsch 1974: 368, Z. 5-7)

(9b) erst einmal möchte ich sagen , + daß ich bedaure,,++ daß ich die einzige der Frauen hier bin + , ,+ die dafür eintreten + ,

(i),+ daß der Paragraph abgeschafft wird,+ .

(ii) $i+$ den Paragraphen abzuschaffen $+\mathrm{i}$.

(iii) ,+ daß man den Paragraphen abschafft + , .

Für die unmittelbare grammatische Umgebung von Nominalisierungen wird gemäß (2) angenommen, daß sie im Vergleich zur unmittelbaren grammatischen 
Umgebung von bedeutungsäquivalenten Nebensätzen weniger markierte Parameterwerte aufweist. Die Ergebnisse in Petrič (1990: 252-313, 1995c: 231-243) scheinen die These weitgehend zu unterstiutzen.

Die Gliedkerne solcher Nominalisierungen sind Verbalabstrakta und können im Vergleich zu den Hauptverben der bedeutungsäquivalenten Nebensätze aufgrund ihrer größeren semantischen und formalen Merkmalhaftigkeit als verstärkte Formen eingeordnet werden. Im Gegensatz zu den zugrundeliegenden Hauptverben gehören sie nicht zu den Default-Ausdrücken des Sprachsystems, werden im kindersprachlichen Lernprozeß relativ spät erworben und sind in der Sprachgeschichte relativ spät entstanden. Default-Ausdruck einer Proposition sind satzartige Konstruktionen, insbesondere solche mit finitem Verb. Das Verb ist jene lexikalische Kategorie, mit der man typischerweise Handlungen und Vorgänge ausdrückt. Wenn Nominalisierungen wie (9a) nicht zu den Default-Ausdrücken einer Proposition gehören, kann man weiterhin vermuten, daß Nominalisierungen aufgrund ihrer informationsverdichtenden Eigenschaften relativ häufig in markierterer "textueller" Umgebung auftreten. Der Sprecher verdichtet Informationen insbesondere in solchen Situationen, in denen hohe Abstraktion des Themas vorliegt und überdurchschnittlich hoher Informationsfluß notwendig ist. Nimmt man ebenenübergreifende Korrespendenzen an (d.h. zwischen der kognitiv-semantischen und textuellen Ebene), könnte daraus folgen, daß die Häufigkeit von Nominalisierungen wie in (9a) gerade in solchen Textsorten zunimmt, die oben als weniger grundlegend, d.h. als markierter eingestuft wurden. Unter den angeführten Grundtextsorten sollte daher insbesondere der schriftliche Fernmonolog (GTS 5) den typischen Erscheinungsort von Nominalisierungen dieses Typs darstellen.

Vergleichen wir zunächst Häufigkeitsdaten aus zwei Dialogen miteinander und anschließend diese Daten mit jenen aus einem schriftlichen Fernmonolog. Die Auszählung der mündlichen Nahdialoge XEG und XAI aus dem Freiburger Korpus (Heutiges Deutsch 1975: 25-42, 87-106) unterstützt die oben formulierte These, daß die Gebrauchshäufigkeit von Nominalisierungen auf <-ung> mit höherem Abstraktionsgrad des Redegegenstandes und größerem Informationsfluß zunimmt. Wahrscheinlich spielt aber auch der verschiedene Öffentlichkeitsgrad der beiden Texte eine Rolle für die verschiedenen Gebrauchsfrequenzen von Nominalisierungen auf <-ung>. Im privat geführten mündlichen Nahdialog XEG (Thema: Gespräch über Familien- und Erziehungsprobleme) beschränkt sich der Anteil der Nominalisierungen auf $0,4 \%$ aller Textwörter, während er im öffentlich geführten mündlichen Nahdialog XAI (Thema: Schulklassengespräch mit Präses Scharf über die Denkschrift der EKD) 2,5\% aller Textwörter ausmacht. Der diesbezügliche Unterschied zwischen den beiden Texten ist statistisch signifikant (Differenzindex DI $=7,3>2$ ). ${ }^{2}$

2 Der Differenzindexwert (DI) zeigt statistische Signifikanz an, wenn der Betrag größer als 2 ist. Zur Berechnung der Differenzindices vgl. Pavlić (1971: 226-229). 


\begin{tabular}{|l|l|l|l|l|l|}
\hline \multicolumn{6}{|c|}{$(\mathbf{1 0 )}$ Vergleich zweier mündlicher Nahdialoge hinsichtlich komplexer Ausdrucksformen } \\
\hline TEXT & Wörter & $\begin{array}{c}\text { Nom. auf } \\
<- \text {-ung }>\end{array}$ & NS mit V-Letzt & NS mit V-2/V-L & NS mit Vi \\
\hline XEG & $3498=100 \%$ & $\begin{array}{l}0,4 \% \\
(\mathrm{DI}=-\mathbf{7 , 3})\end{array}$ & $\begin{array}{l}24,2 \% \\
(\mathrm{DI}=-3,0)\end{array}$ & $\begin{array}{l}11,3 \% \\
(\mathrm{DI}=\mathbf{3 , 0})\end{array}$ & $\begin{array}{l}2,8 \% \\
(\mathrm{DI}=1,9)\end{array}$ \\
\hline XAI & $4484=100 \%$ & $2,5 \%$ & $33,6 \%$ & $5,6 \%$ & $5,4 \%$ \\
\hline
\end{tabular}

Den größeren Abstraktionsgrad des Redegegenstandes von XAI scheint übrigens auch der größere Anteil der V-Letzt-Nebensätze anzuzeigen. Im Text XAI beträgt der Anteil der V-Letzt-Nebensätze 33,6\%, im Text XEG hingegen nur 24,2\%. Der diesbezügliche Unterschied zwischen den beiden Stichproben ist statistisch signifikant (Differenzindex DI = 3,0>2). Entsprechendes gilt wohl auch für den Anteil der Infinitivsätze, da der Unterschied zwischen XEG und XAI (aufgrund der kleineren Stichproben) nur knapp unter der Signifikanzgrenze liegt $(2,8 \%: 5,4 \%$; DI $=1,9<2)$. Der Anteil der abhängigen V-2-Hauptsätze ist hingegen im weniger abstrakten Text XEG größer als im abstrakteren XAI $(11,3 \%: 5,6 \%$; DI =3,0 > 2). Dies zeugt von einem größeren Anteil von Nebensätzen, die von den weniger markierten Verben des Sagens und Denkens u.ä. abhängen.

Die Auszählung des relativ abstrakten mündlichen Nahdialogs XAI und des relativ abstrakten schriftlichen Fernmonologs GG (Grundgesetz für die Bundesrepublik Deutschland 1993: 12-91) nach Nominalisierungen auf <-ung> ergibt folgendes Bild:

\begin{tabular}{|l|l|l|}
\hline \multicolumn{3}{|c|}{ (11) Vergleich zweier mündlicher Nahdialoge hinsichtl. Nominalisierungen } \\
\hline TEXT & Wörter & Nominalisierung auf <-ung> \\
\hline GG & $18237=100 \%$ & $874=4,8 \%($ DI = 6,8 > 2) \\
\hline XAI & $4484=100 \%$ & $112=2,5 \%$ \\
\hline
\end{tabular}

Der Anteil von Nominalisierungen auf <-ung > ist im schriftlichen Ferndialog GG signifikant größer als im mündlichen Nahdialog XAI. Die angeführten Daten bestätigen die Vermutung, daß unter den angeführten Grundtextsorten insbesondere der schriftliche Fernmonolog (GTS 5) den typischen Erscheinungsort von Nominalisierungen des oben ausgezählten Typs darstellt. Oder anders ausgedrückt: Syntaktische Simplifizierungen mit Hilfe von Nominalisierungen als Nicht-Default-Ausdrücken von Propositionen scheinen häufiger in markierteren Textsorten aufzutreten.

\subsection{Variantenpaar 2: Funktionsverbgefüge vs. Verb}

Ein Funktionsverbgefüge (12a) - zur Abgrenzung von freien Fügungen und Nominalisierungsverbgefügen siehe v. Polenz (1987: 170) und So (1991: 11-13), - ist im Vergleich $\mathrm{zu}$ einem bedeutungsäquivalenten Verb (12b) eine verstärkte 
syntaktische Konstruktion (vgl. Petrič 1990: 122-131, 1993b: 128-136, 1994: 190-194).

(12a) Der Zug kommt in Bewegung.

(12b) Der Zug bewegt sich.

Funktionsverbgefüge sind prinzipiell formal aufwendiger und semantisch spezifischer als die entsprechenden bedeutungsäquivalenten Verben (vgl. z.B. bewegen mit in Bewegung kommen). Gemäß (1) bedeuten sie daher eine Komplikation für den Textproduzenten und eine Dekodierungserleichterung für den Textrezipienten. Funktionsverbgefüge gehören außerdem nicht zu den Default-Ausdrücken für Handlungen und Vorgänge im Sprachsystem, denn die Handlung oder der Vorgang werden nicht verbal, sondern nominal ausgedrückt (d.h. meist mit einem Verbalabstraktum). Diese sprachliche Variante wird im kindersprachlichen Lernprozeß relativ spät erworben und entwickelte sich im Deutschen und in jenen Sprachen, in denen vergleichbare Konstruktionen bereitstehen, später als die verbale Ausdrucksweise. Geht man von den angeführten Eigenschaften aus, scheint gemäß (1) die Wahrscheinlichkeit gegeben, daß sich Funktionsverbgefüge im Vergleich zu den bedeutungsäquivalenten Verben vorzugweise in markierter grammatischer Umgebung (Satzebene) und insbesondere im schriftlichen Fernmonolog (Textebene) behaupten.

In einem Korpus, das aus 15 Diskussionen (Heutiges Deutsch 1971 und 1974 sowie Protokoll eines Werkstattgesprächs 1971: 16-39) besteht, wurden Funktionsverbgefüge und Nominalisierungsverbgefüge ausgezählt. Es wurden folgende Zahlenverhältnisse festgestellt: Funktionsverbgefüge mit Satzgliedcharakter sind in 113 Haupt- und Nebensätzen sowie 13 Infinitivsätzen $\mathrm{zu}$ finden, Nominalisierungsverbgefüge mit Satzgliedcharakter hingegen in 427 Haupt- und Nebensätzen sowie 27 Infinitivsätzen. Die Ergebnisse in Petrič (1994: 136-144) scheinen die These weitgehend zu unterstützen, daß der Kontext von Funktionsverbgefügen im Vergleich zum Kontext der Hauptverben im Kontrollsample markiertere Parameter aufweist. Die oben angeführten Zahlen zeigen auch, wie selten Funktionsverbgefüge oder Nominalisierungsverbgefüge in mündlichen Nahdialogen auftreten. Der Anteil von Funktionsverbgefügen oder Nominalisierungsverbgefügen in schriftlichen Fernmonologen sollte gemäß der oben geäußerten Vermutung größer sein. Einen Hinweis auf die Richtigkeit der These bietet das Korpus von So (1991). Bei einem Vergleich der Anteile der Funktionsverbgefüge und der Nominalisierungsverbgefüge am Prädikatsausdruck zeigte sich folgendes: Während der Anteil der Funktionsverbgefüge bzw. der Nominalisierungsverbgefüge in 12 mündlichen Nahdialogen aus dem Freiburger Korpus (Heutiges Deutsch 1974) bei etwa $1,7 \%$ bzw. 6,3\% liegt, beträgt der Anteil der Funktionsverbgefüge bzw. der Nominalisierungsverbgefüge in den 5 Texten aus dem 20. Jahrhundert, die So (1991: 160-199) als Datenbasis verwendet und die zur Grundtextsorte schriftlicher Fernmonolog gehören, etwa 2,7\% bzw. 7,4\%. Die Unterschiede zwischen den 
miteinander verglichenen mündlichen Nahdialogen und den schriftlichen Fernmonologen sind statistisch signifikant (DI = 4,8 bzw. DI =3,3).

Die Frage, ob Funktionsverbgefüge dem Textproduzenten wirklich mehr Schwierigkeiten bereiten als die bedeutungsäquivalenten Verben, läßt sich nicht immer zweifelsfrei beantworten. Die als markiert geltenden Passivkonstruktionen (13b) können durch die ebenfalls als markiert bewerteten Funktionsverbgefüge (13a) ersetzt werden.

(13a) die Tatsache $+\mathrm{k}$ daß ich für ( $k a n n$ ich vielleicht darauf eingehen ) die Tatsache ,+ daß verhältnismäßig wenig $+g+$ derartiger Straftaten zur Anzeige und Aburteilung kommen + , macht keineswegs die Strafvorschrift unnötig. (Heutiges Deutsch 1974: 378)

(13b) die Tatsache $+\mathrm{k}$ daß ich für ( $k a n n$ ich vielleicht darauf eingehen ) die Tatsache ,+ daß verhältnismäßig wenig $+g+$ derartiger Straftaten angezeigt und abgeurteilt werden + , macht keineswegs die Strafvorschrift unnötig.

Gegenüber Passivkonstruktionen haben Funktionsverbgefüge wohl prinzipiell den Vorteil, als Ganzes (ähnlich wie ein Phraseologismus) aus dem Lexikon abrufbar zu sein, während Passivkonstruktionen (vermutlich) erst speziell für diesen Kontext neu gebildet werden müssen. Die ganzheitliche Verarbeitung von Information bringt durchaus eine Erleichterung für den Textproduzenten als auch für den Textrezipienten mit sich. Auf dieser Grundlage könnten Passivkonstruktionen im Vergleich zu den entsprechenden Funktionsverbgefügen als markierter eingestuft werden.

\subsection{Variantenpaar 3: W-Passiv vs. Aktiv}

Die Default-Ausdruckskategorie für die Geschehensperspektive ist das Aktiv (14b). Das Werden-Passiv (14a) ist in dieser Hinsicht markiert, denn die semantischen Rollen Agens und Patiens sind im Passivsatz nicht so serialisiert, wie das für Nominativ-Akkusativ-Sprachen typisch ist (vgl. dazu Leiss 1992: 149ff.). In den Nominativ-Akkusativ-Sprachen ist die Abfolge Subjekt vor Akkusativ grundlegend. Das prototypische Subjekt in solchen Sprachen ist Agens, belebt und thematisch, das prototypische Akkusativobjekt (direktes Objekt) in solchen Sprachen ist Patiens, unbelebt und rhematisch. Wenn ein Sachverhalt mit thematischem, unbelebtem Patiens versprachlicht wird, wird in Sprachen mit relativ starren Abfolgeregeln, z.B. im Englischen oder Deutschen, eine passivische Ausdrucksweise bevorzugt, d.h. das definite, unbelebte Patiens wird als Subjekt realisiert und damit in der syntaktischen Position des direkten Objekts vermieden. Auf kognitiv-semantischer Ebene könnte die Passiv-Perspektive eine Erschwernis sowohl für den Sprecher als auch für den Hörer bedeuten, insbesondere aber für den letzteren, der seine Erwartungen hinsichtlich der Geschehensperspektive an die vom Sprecher realisierte anpassen muß.

(14a) Viele Frauen sind der Meinung, daß der Paragraph abgeschafft werden sollte. [vom Gesetzgeber?] 
(14b) Viele Frauen sind der Meinung, daß der Gesetzgeber den Paragraphen abschaffen sollte.

Als Konstruktionstyp ist das Werden-Passiv eine weniger prototypische Ausdrucksweise für Handlungen oder Vorgänge, denn das Hauptverb wird aus der Rolle des finiten Verbs verdrängt und als Partizip ausgedrückt (d.h. nominaler).

Die mögliche, aber selten genutzte Vollform eines Satzes mit Werden-Passiv (d.h. mit Agensangabe) ist formal komplexer als die eines Aktivsatzes. Im Textgebrauch ist ein Satz mit dem Werden-Passiv einerseits merkmalloser als ein Aktivsatz, denn die Angabe des Agens ist im Passivsatz - im Gegensatz zum Aktivsatz - fakultativ und fehlt in den meisten Fällen (wie bei den Nominalisierungen - vgl. (9a) mit (9b)). Andererseits ist das Werden-Passiv durch die Hinzufügung des Auxiliarverbs werden im verbalen Bereich merkmalhafter als das Aktiv. Auf Artikulationsebene bedeutet das Passiv mit nicht-realisierter Agensphrase und hinzugefügtem Auxiliarverb meist eine geringfügige Erleichterung für den Sprecher. Der größere Vorteil liegt aber wohl auf kognitiv-semantischer Ebene, denn der Sprecher braucht die vorausgesetzte Agensphrase nicht erneut aus dem Lexikon abzurufen. Ein Passivsatz ist wie eine Nominalisierung semantisch vager. Die Ermittlung der (meist) ausgesparten Agensangabe stellt eine Schwierigkeit für den Textrezipienten dar, insbesondere dann, wenn im Kontext nicht genügend Hinweise im Hinblick auf sein (lüickenhaftes) Weltwissen vorhanden sind. Allerdings bringt das Passiv dem Hörer auch eine Erleichterung im Hinblick auf die Thema-Rhema-Gliederung des Satzes, denn durch das Passiv wird die üblichere Anordnung Thema vor Rhema wiederhergestellt. Liegt nämlich ein thematisches direktes Objekt vor, kann der Sprecher die vom Hörer erwartete Abfolge Thema vor Rhema realisieren. Passivsätze werden im allgemeinen später erlernt als Aktivsätze. ${ }^{3}$

In den Arbeiten von Teržan-Kopecky finden sich mehrere Anhaltspunkte für die Angemessenheit der Behauptung, daß das Werden-Passiv als verstärkte Konstruktion in Kontexten mit markierteren Parameterwerten auftritt (vgl. z.B. Teržan 1990: 93-97). Die Behauptung, daß das Werden-Passiv als verstärkte syntaktische Konstruktion besonders typisch im schriftlichen Fernmonolog ist, wird durch statistische Daten in Brinker (1971: 68) unterstützt. Im Mischkorpus IDS3 (Heutiges Deutsch 1975), d.h. einer Textsammlung mit hauptsächlich weniger markierten mündlichen Nahdialogen, scheint der Anteil des Werden-Passivs geringer zu sein als im Korpus IDS2 (Heutiges Deutsch 1974), das einheitlich aus markierteren mündlichen Nahdialogen Diskussionen - besteht, sowie geringer als im Grundgesetz der Bundesrepublik Deutschland (Ausgabe 1993: 12-91), denn der Anteil des Lexems werden bezüglich aller Textwörter beträgt 0,4\% im Mischkorpus IDS3, 0,7\% im Diskussionskorpus IDS2

3 Zwischen den Passivsätzen bestehen hinsichtlich ihrer Komplexität auch andere Unterschiede. Bekannt ist z.B., daß Kinder im Spracherwerb eher solche Passivsätze erlernen, in denen das Patiens im Kontrast zum Agens eine nicht-belebte Größe darstellt, und später solche, in denen das Patiens wie das Agens eine belebte Größe darstellt (Schwarz $\left.{ }^{2} 1996: 134\right)$. 
und 0,6\% im Grundgesetz. Der Unterschied zwischen dem Mischkorpus IDS3 und den markierteren Textsorten in IDS2 und im Grundgesetz ist statistisch signifikant (DI = 5,38 bzw. 3,21).

\subsection{Variantenpaar 4: Futur vs. Präsens pro futuro}

Die Default-Ausdruckskategorie für den Bezug auf Zukünftiges ist im Deutschen nicht das Futur werden + Infinitiv (15a), sondern das Präsens pro futuro (15b). Als Konstruktionstyp ist das Werden-Futur eine weniger prototypische Ausdrucksweise für Handlungen oder Vorgänge, denn das Hauptverb wird aus der Rolle des finiten Verbs verdrängt und als Inifinitiv ausgedrückt (d.h. nominaler). Von 2000 zukunftsbezogenen Sätzen im Korpus von Brons-Albert entfallen 76,0\% auf Sätze mit Präsens und lediglich 4,6\% auf Sätze mit werden + Infinitiv (Brons-Albert 1982: 43).

(15a) A: Aber es soll gut sein ! ((lacht))

B: Na gut, dann werd ich mal versuchen, es zu bekommen, ne. Ne?

A: Ja. Dann wirst du mal en paar Buchhändler verrückt machen !

B: Ach, ich kann ja keinen verrückt machen, ich mach das auf die ruhige Art !

A: Du has ein werden vergessen!

B: ((lacht)) Nein, $d$ / das weiß ich, ne. Aber das is mir zuviel, das immer zu sagen.

(Brons Albert 1982: 81)

(15b) A: Ja. Dann wirst du mal en paar Buchhändler verrïckt machen !

B: Ach, ich kann ja keinen verrïckt machen, ich mach das auf die ruhige Art !

Das deutsche Futur ist durch die Hinzufügung eines speziellen Auxiliarverbs merkmalhafter als das Präsens, außerdem wird das Hauptverb aus der (prototypischeren) Rolle des finiten Verbs verdrängt und als Infinitiv realisiert, d.h. in einer nominaleren Verbform. Relativ oft verlangt das Präsens für den Zukunftsbezug allerdings kompensierende situierende Ausdrucksmittel im umgebenden Satz, im weiteren Kontext oder im Situationszusammenhang, damit der Zukunftsbezug gewährleistet ist (vgl. dazu Brons-Albert 1982: 49; Teržan-Kopecky 1995: 237-238). Doch selbst das werden-Futur leistet oft keinen eindeutigen Zukunftsbezug, was wohl an der nicht seltenen Verbindung mit Zeitadverbialen sichtbar wird (Brons-Albert 1982: 102). Das (kataphorische) Futur hat sich als Tempus relativ spät herausgebildet (vgl. Saltveit 1962: 175). Sein typischer Erscheinungsort ist der schriftliche Fernmonolog. Daten aus Gelhaus (1975: 23-24) zeigen, daß der Anteil des werden-Futurs in schriftlichen Fernmonologen starken Schwankungen unterworfen ist, d.h. von $0,4 \%$ bis $6,0 \%$ aller in den Texten vertretenen Tempusformen. Im Korpus von Gelhaus zeichnen sich insbesondere die Zeitungen und Zeitschriften durch relativ höhere Futur-Anteile aus (1,9\%-6,0), während die im Korpus vertretenen Dramen relativ geringere Futur-Anteile aufweisen (2,1\%-2,7\%). Die eben angeführten Daten könnten ein Anhaltspunkt dafür sein, daß die werden-Konstruktion für den Bezug auf 
Zukünftiges in weniger markierten mündlichen Nahdialogen seltener vorkommen als in markierteren schriftlichen Fernmonologen. Zu einem ähnlichen Schluß kommt auch Brons-Albert (1982: 100), die wie Dittmann (1976: 235) im Freiburger Korpus die starke Textsortenabhängigkeit des werden-Futurs hervorhebt.

\subsection{Variantenpaar 5: Perfekt vs. Präteritum}

Das Präteritum ist formal weniger salient als das Perfekt, denn der Vergangenheitsbezug wird nicht wie beim Perfekt durch die Kombination silbischer Morpheme und eines nicht nicht-silbischen Morphems (z.B. haben + ge- $-t$ in hat gekauft) geleistet, sondern lediglich durch ein silbisches (-te, z.B. in kaufte) oder sogar nur durch ein nicht-silbisches (Ablaut, z.B. in rief von rufen). Das Perfekt könnte demnach aufgrund seiner formalen Merkmalhaftigkeit als starke Variante angesehen werden. Hinweis für die Angemessenheit dieser These ist laut Orešnik (1996: 3-10) das Auftreten des Perfekts mit einer Reihe von markierteren Parameterwerten (im Vergleich zum Präteritum: z.B. häufiger mit nicht-dritten Personen, seltenere Verbindung mit sehr häufig auftretenden Hauptverben, vergleichsweise seltenes Auftreten in intransitiven Sein-Konstruktionen, selteneres Erscheinen in den als unmarkiert angesehenen Relativsätzen, häufigeres Auftreten in Fragesätzen). Als Konstruktionstyp kann man das Perfekt außerdem als weniger prototypische Ausdrucksweise für Handlungen oder Vorgänge einordnen, denn das Hauptverb wird aus der Rolle des finiten Verbs verdrängt und als Partizip ausgedrückt (d.h. nominaler).

(16a) B: Also, Kinder, machts gut, bleibt brav, besauft euch nich /

A: Du dich auch nicht!

B: Ne, was meinsde, was das in Finnland kostet!

A: Jaja, aber diese Feste in Finnland, von denen wir gehört haben, wie du das erzählt has, man säuft erst so viel, daß man nich mehr gehen kann / (Brons Albert 1982: 10)

(16b) A: Jaja, aber diese Feste in Finnland, von denen wir hörten, wie du das erzähltest, man säuft erst so viel, daß man nich mehr gehen kann /

Die Einordnung der beiden Vergangenheitstempora Perfekt und Präteritum als starke bzw. schwache syntaktische Konstruktion ist trotzdem nicht unproblematisch. Laut Grundannahme weist die starke Variante im Variantenstadium gewöhnlich einen höheren Informationsgehalt (d.h. eine komplexere Semantik) auf oder unterscheidet sich von der schwachen Variante zumindest durch stilistische Besonderheiten bzw. Beschränkungen. Das analytische Perfekt und das synthetische Präteritum sind annähernd synonyme Ausdrücke für den Bezug auf die Vergangenheitssphäre. Die Substitutionsmöglichkeiten sind eingeschränkt, denn bei Außerachtlassung stilistischer Unterschiede kann zwar ein Präteritum durch ein Perfekt ersetzt werden, aber nicht jedes Perfekt durch ein Präteritum (vgl. Hentschel/Weydt 21994: 100). Perfekt und Präteritum als Vergangenheitstempora unterscheiden sich hinsichtlich des semantischen Merkmals der zeitlichen Begrenzung oder Abgeschlossenheit (vgl. Sieh mal, es hat 
geschneit mit *Sieh mal, es schneite). Das Perfekt weist den als markierter angenommen Parameterwert der Begrenzung auf, das Präteritum dagegen den als weniger markiert angenommenen Parameterwert der nichtspezifizierten Begrenzung (vgl. Es schneite mit Es hat geschneit). Dies steht in Einklang mit der Annahme in (1), daß das Perfekt als starke Variante einen höheren Informationsgehalt aufweist. Auffällig ist jedoch, daß gerade das als schwache Variante bezeichnete Präteritum stilistische Beschränkungen zeigt (d.h. speziellere Gebrauchsbedingungen fordert als das mutmaßlich starke Perfekt) und daß es eine relativ klare Affinität zu monologischen Texten zeigt, insbesondere zu schriftlichen Fernmonologen, und zwar vor allem in solchen, in denen die Aufmerksamkeit des Lesers auf die Abfolge der dargestellten Ereignisse in der Vergangenheit gerichtet wird (vgl. Latzel 1977). Dieser Umstand scheint in Widerspruch zur Annahme in (4) und (5) zu stehen.

\subsection{Variantenpaar 6: Modalpartikelhaltiger Satz vs. modalpartikelloser Satz}

Sätze mit Modalpartikeln (Abtönungspartikeln) können in synchronischer Sichtweise als starke Varianten modalpartikelloser Sätze mit derselben Proposition gewertet werden.

(17a) A: Tag, B, hier is A. Hör mal, ich wollt dich fragen, mußt du denn nich zum Flughafen gebracht werden und abgeholt werden?

B: Eh, nein, diesmal nicht, weil ich ja nicht wieder zum Flughafen zurückkomme.

A: Ah so.

B: Nich, ich werd ja, ich fahr ja mit dem Z zurück. (Brons Albert 1982: 1)

(17b) A: Tag, B, hier is A. Hör, ich wollt dich fragen, mußt du zum Flughafen gebracht werden und abgeholt werden?

B: Eh, nein, diesmal nicht, weil ich nicht wieder zum Flughafen zurückkomme.

A: Ah so.

B: Nich, ich werd, ich fahr mit dem Z zurück.

Die deutschen Modalpartikeln (MPn) an sich sind einfache Wörter. Die prototypischen unter ihnen sind einsilbig, Simplizia, häufig vorkommende Wörter mit sehr allgemeinen denotativen Bedeutungskomponenten und vorherrschenden konnotativen Bedeutungsanteilen, im Satz unbetont, nicht isolierbar und nicht erststellenfähig. Von einigen Modalpartikeln (z.B. mal und denn - vgl. Lindner 1983: $211 \mathrm{ff}$.) ist bekannt, daß sie von deutschsprachigen Kindern bereits vor dem vierten Lebensjahr erworben werden.

Ich nehme an, daß die MPn zuerst als Gebrauchsvariante formal einfacher Wörter mit allgemeiner denotativer Bedeutung entstanden (z.B. ja, doch, denn), daß sich jedoch in einem späteren Zeitabschnitt des Neuhochdeutschen auch MPn als Variante formal relativ aufwendiger Wörter einstellten (z.B. eigentlich, sowieso, vielleicht). Historisch gesehen ist laut Grundannahme wahrscheinlich, daß die heutigen MPn als 
schwache Gebrauchsvarianten autosemantischer Ausdrücke zunächst in einfacheren grammatischen Umgebungen entstanden, nun aber in den Sätzen, in denen sie auftreten, als modalitätsverstärkende Elemente wirken.

Bei der Bewertung, ob modalpartikelhaltige Sätze im Vergleich zu modalpartikellosen Sätzen starke Varianten sind, berücksichtige ich die folgenden Kriterien: 1. den formalen Aufwand, 2. den Informationsgehalt, 3. die Sprecherfreundlichkeit, 4. die Hörerfreundlichkeit der zu vergleichenden Konstruktionen. Zusammenfassend läßt sich behaupten:

1. Ein MP-haltiger Satz ist nicht so zeit- oder platzsparend wie ein MP-loser Satz, denn er enthält eine zusätzliche syntaktische Stelle (d.h. die eines Adjunkts). Laut Krivonosov (1977: 188ff.) korreliert der Gebrauch einer Modalpartikel auch mit einer Anhebung der Grundfrequenz, die dem modalpartikelhaltigen Satz emphatischer erscheinen läßt.

2. Ein MP-haltiger Satz hat im Vergleich zu einem MP-losen Satz einen größeren oder zumindest eindeutiger bestimmbaren Informationsgehalt, denn durch das Hinzufügen einer MP signalisiert der Sprecher eindeutiger als mit anderen Mitteln (insbesondere dem Satzmodus und dem Intonationsmuster), welche Einstellung er gegenüber einem Satzinhalt (Proposition) einnimmt. In einigen Fällen wird erst durch Modalpartikeln deutlich, wie der Hörer den Satzinhalt einer Äußerung zu verstehen hat und welche verbalen oder nicht-verbalen Reaktionsmöglichkeiten sich für ihn eröffnen. Durch die Hinzufügung einer MP ist es dem Sprecher demnach möglich, den kommunikativen Sinn einer $\ddot{A} u ß e r u n g ~ z u$ verdeutlichen und den Fortgang von Gesprächssequenzen (das Fortsetzungsraster) unbewußt zu beeinflussen. Einzelsprachlich gesehen handelt es sich bei den Modalpartikeln um eine prototypische Ausdrucksweise für situative Verweise. Universell gesehen ist es allerdings weniger typisch, daß ein derartiger situativer Verweis mit speziellen Lexemen vorgenommen wird, denn üblicherweise geschieht dies mit Hilfe des Satzmodus und des Intonationsmusters.

3. Laut Grundannahme bereiten starke Varianten dem Sprecher größere Schwierigkeiten als schwache Varianten. Die Beurteilung der Sprecherfreundlichkeit von MP-haltigen Sätzen ist schwierig, weil empirisch relativ wenig darüber bekannt ist und wir vor allem auf Hypothesen angewiesen sind. Ein wichtiger Angelpunkt meiner Bewertung ist der (leichter nachweisbare) formale Aufwand. Da ein MP-haltiger Satz formal aufwendiger ist als ein MP-loser (siehe oben), könnte man davon ausgehen, daß er dem Sprecher größere Schwierigkeiten bereitet. Allerdings ist der formale Mehr-Aufwand relativ gering, weil MP meist einsilbige und unbetonte Lexeme sind. Die in Texten feststellbare Gebrauchshäufigkeit der MPn ist zwar vergleichsweise gering (vgl. Hentschel 1986: 238ff., Petrič 1995d: 119-121), die den MPn zugrundeliegenden (etymologisch verwandten) Wörter gehören allerdings laut Frequenzwörterbüchern zu den häufigeren deutschen Lexemen (Meier 1978). Hypothetischer ist die Beurteilung des Aufwandes auf semantisch-kognitiver Ebene. Aus der Tatsache, daß es ganz spezifische Gebrauchssituationen gibt, in denen eine 
bestimmte MP verwendet, und solche, in denen eine bestimmte MP nicht verwendet werden kann, schließe ich, daß die Auswahl einer dem kommunikativen Sinn entsprechenden MP dem Sprecher Schwierigkeiten bereitet. Obwohl einige MPn nachweisbar früh erworben werden (siehe oben), scheint mir außerdem wahrscheinlich, daß die konnotativen Bedeutungskomponeneten der meisten MPn erst in späteren Phasen der Sozialisierung durch positive Evidenz gelernt und vor allem die grundlegenden denotativen Bedeutungskomponenten der MPn durch den Erwerb der ihnen zugrundeliegenden Wörter relativ früh gelernt werden. MPn gehören zu jenen sprachlichen Elementen, die unbewußt realisiert werden.

4. Dem Hörer erleichtert die MP das Verständnis des kommunikativen Sinnes der Äußerung. Eine MP stellt ein zusätzliches Signal oder in bestimmten Fällen sogar das deutlichste Signal für die Dekodierung des kommunikativen Sinnes einer Äußerung dar. Allerdings ließe sich einwenden, daß MPn aufgrund ihrer knappen Form ziemlich unauffällige (wenig saliente) sprachliche Elemente sind und damit den gebundenen Morphemen schon recht nahe kommen. Das scheint auch daraus zu folgen, daß sich sowohl Sprecher als auch Hörer nicht bewußt sind, welche MP in einem Satz verwendet wurde, wenn sie die Aufgabe haben, einen Satz vollständig zu wiederholen. MPn werden im Falle von Satzwiederholungen regelmäßig ausgelassen.

Trotz einiger oben angeführter möglicher Einwände ist es m. E. sinnvoll, MP-haltige Sätze als starke Varianten MP-loser Sätze zu betrachten.

Wie empirische Untersuchungen zeigen, ist der typische Erscheinungsort der MPn in mündlichen Nahdialogen zu suchen, insbesondere in solchen, die einen hohen Privatheitsgrad aufweisen (vgl. Hentschel 1986: 238ff., Petrič 1995d: 119-121). Im Gegensatz zu den anderen oben behandelten verstärkten Konstruktionen dienen die MPn nicht zur Verdeutlichung bestimmter denotativer Bedeutungskomponenten in der Proposition einer Äußerung, sondern vor allem als verdeutlichende (zusätzliche) Signale für bestimmte konnotative Bedeutungskomponenten, die die Beurteilung der Proposition betreffen (z.B. die MP eben die Einstellung, daß die Proposition einer Äußerung vom Sprecher als evidente Begründung für einen zuvor eingetretenen Umstand eingeordnet wird) und die meist auch vom Satzmodus und dem Intonationsmuster in rudimentärer Form angezeigt werden (können). Dies hat zur Folge, daß MPn vor allem in jenen Textsorten häufiger auftreten, in denen diesbezügliche Beziehungsaspekte zwischen den Kommunikationsteilnehmern im Vordergrund und präzise Beschreibungen und Argumentationen im Hintergrund stehen, d.h. in mündlichen Nahdialogen mit hohem Privatheitsgrad. Nach meiner bisher vertretenen Auffassung könnten denotative Bedeutungsaspekte ausschlaggebend für die textsortenspezifische Distribution von starken und schwachen Varianten sein. MPn sind, was ihre denotativen Bedeutungskomponenten anbelangt, schwache Varianten der ihnen zugrundeliegenden Wörter. Von daher scheint es mir nicht verwunderlich, daß sie in weniger markierten Grundtextsorten häufiger auftreten als in markierten. 
Aus einer alternativen Perspektive lassen sich MP-haltige Sätze als schwache Varianten MP-loser Sätze samt Äußerungssituation beschreiben (Petrič 1995a: 285). MPn wären demnach auffaßbar als kondensierte sprachliche Ausdrücke mit der Funktion des Verweises auf die sprachliche Umgebung und die Situation, d.h. ein Lexem, das bestimmte Szenarios aktualisiert. Diese Anschauung beruht auf der Vorstellung, daß man isolierten MP-haltigen Sätzen aufgrund der MP-Semantik bestimmte Kontexttypen und Typen von Äußerungssituationen zuordnen kann, in denen sie vorkommen können. Aus dieser Perspektive ließen sich folgende Behauptungen aufstellen:

1. MP-haltige Sätze sind im Vergleich zu MP-losen Sätzen + Kontext + Situation schwache Varianten, weil sie formal weniger aufwendig sind. MPn treten daher recht häufig in konventionalisierten syntaktischen Strukturen, sprachlichen Routinen und phraseologischen Prägungen auf.

2. MP-haltige Sätze sind im Vergleich zu MP-losen Sätzen + Kontext + Situation schwache Varianten, weil sie einen geringeren Informationsgehalt aufweisen. In einem ein- oder zweisilbigen Lexem lassen sich verständlicherweise nicht so viele Informationen unterbringen wie in einem ganzen Satz und in der ihn umgebenden Situation.

3. MP-haltige Sätze sind im Vergleich zu MP-losen Sätzen + Kontext + Situation schwache Varianten, weil sie vom Sprecher leichter zu realisieren sind. Die Produktion eines entsprechenden Kontextes und der Aufbau einer bestimmten Situation, die den Sinn einer Äußerung verständlich machen, ist schwieriger als die Produktion eines entsprechenden einzelnen Lexems.

4. MP-haltige Sätze sind im Vergleich zu MP-losen Sätzen + Kontext + Situation schwache Varianten, weil sie vom Hörer nicht so leicht zu dekodieren sind. Daß MP-Bedeutungen nicht so einfach zu verstehen sind, zeigt sich insbesondere im Fremdsprachenunterricht.

Die Einordnung MP-haltiger Sätze als schwacher Varianten im Vergleich zu MP-losen Sätzen + Kontext + Situation wäre auch vereinbar mit der Annahme (5), nach der schwache Varianten häufiger in weniger markierten Grundtextsorten auftreten, d.h. in mündlichen Nahdialogen. MP-haltige Sätze als schwache Varianten kämen vor allem in solchen Texten vor, in denen ein expliziter Bezugskontext oder eine explizite Bezugssituation häufig fehlt. Möglicherweise sind gerade mündliche Nahdialoge mit hohem Privatheitsgrad, für die aufgrund der Personen- und Situationsvertrautheit elliptische Kontexte durchaus typisch sind, solche Texte.

Ein grundlegendes Problem der Annahme, MP-haltige Sätze seien schwache Varianten, ist allerdings, daß sie nicht in allen Einzelheiten empirisch überprüfbar zu sein scheint wie die oben angeführte, nach der MP-haltige Sätze als starke Varianten MP-loser Sätze eingeordnet wurden. Hypothetische Kontexte und Situationen entziehen sich nämlich einer empirischen Untersuchung weitgehend. Ein weiteres Problem liegt darin, daß auch MP-haltige Sätze (wie MP-lose Sätze) gewöhnlich nicht isoliert vorkommen, sondern in bestimmten Kontext- und Situationstypen eingebettet 
sind. Mit der Annahme, MP-haltige Sätze seien schwache Varianten zu MP-losen Sätzen mit Kontext und Situation, wird aber gerade davon ausgegangen.

\subsection{Personalpronomina vs. Nominalphrasen}

Personalpronomina (d.h. er, sie, es) und Nominalphrasen sind paradigmatische Varianten im weiteren Sinne. Ein Personalpronomen wiederholt wie andere anaphorische Ausdrücke nur so viele Merkmale des Nomens (und der übrigen Nominalphrase), wie zur Herstellung eines eindeutigen Bezugs notwendig sind, d.h. Numerus und Genus.

(18a) Sie sahen ihn schon von weitem. Er hatte ein ganz altes Gesicht, aber an seinem Gang konnte man erkennen, daß er jung war.

(18b) Die Leute sahen den Mann schon von weitem. Der Mann hatte ein ganz altes Gesicht, aber an seinem Gang konnte jeder Mensch erkennen, daß der Mann jung war.

Personalpronomina sind merkmallosere (denotativ schwächere) Ausdrücke als Nominalphrasen und weisen vorwiegend phorische Bedeutung auf. Demnach erwarte ich, daß sie in markierteren Textsorten seltener auftreten, d.h. seltener im schriftlichen Fernmonolog.

Der Vergleich zweier mündlicher Nahdialoge aus dem Freiburger Korpus (Heutiges Deutsch 1975) zeigt, daß aufgrund von Abstraktionsunterschieden zwischen Texten bereits ein Variantengefälle vorliegen kann. Im Text XEG ("Smalltalk") werden konkretere Themen besprochen als im Text XAI ("öffentliche Diskussion"). Der größere Anteil der Personalpronomina im Text XEG korreliert übrigens auch mit einem geringeren Anteil an Nomina auf -ung (siehe oben), die den Abstraktionsgrad eines Textes recht deutlich anzeigen.

(19) Anteil der Personalpronomina in den Texten XEG und XAI des Freiburger Korpus

\begin{tabular}{|l|l|l|}
\hline & XEG & XAI \\
\hline er + ihn & 40 & 5 \\
\hline sie & 13 & 15 \\
\hline es & 48 & 50 \\
\hline sie & 5 & 9 \\
\hline Summe der Personalpronomina & 106 & 79 \\
\hline Summe aller Textwörter & 3498 & 4484 \\
\hline Anteil der Personalpronomina \% & 3,03 & 1,76 \\
\hline signifikanter Unter: XEG $>$ XAI DI $=\mathbf{3 , 7 4}>\mathbf{2}$ & & \\
\hline
\end{tabular}

Ein Vergleich des mündlichen Nahdialogs XAI (einer öffentlichen Diskussion) und des schriftlichen Fernmonologs GG (Grundgesetz 1993, Rechtstext) offenbart, daß 
der Anteil der Personalpronomina im schriftlichen Fernmonolog wesentlicher geringer ist als im mündlichen Nahdialog, obwohl in beiden Texten abstrakte Themen behandelt werden. Juristische Texte sind in dieser Hinsicht unter den schriftlichen Fernmonologen extreme Beispiele (vgl. Brandt 1988: 108ff.).

\begin{tabular}{|l|l|l|}
\hline \multicolumn{3}{|l|}{ (20) Anteil der Personalpronomina im Grundgesetz (GG) und in XAI (Freibg. K.) } \\
\hline & GG & XAI \\
\hline er + ihn & 24 & 5 \\
\hline sie (Sg. + Pl.) & 49 & 24 \\
\hline es & 51 & 50 \\
\hline Summe der Personalpronomina & 124 & 79 \\
\hline Summe aller Textwörter & 18237 & 4484 \\
\hline Anteil der Personalpronomina \% & 0,68 & 1,76 \\
\hline signifikanter Unter.: GG < XAI DI = $\mathbf{6 , 9 0}>\mathbf{2}$ & \\
\hline
\end{tabular}

\subsection{Obersatz mit satzförmiger Konstituente vs. Obersatz mit nicht-satzförmiger Konstituente}

Ein Obersatz (d.h. ein übergeordneter Haupt- oder Nebensatz) mit satzförmiger Konstituente (d.h. mit abhängigem Hauptsatz, Nebensatz oder Infinitivsatz) ist im Vergleich zu einem Obersatz mit nicht-satzförmiger Konstituente (d.h. einer Nominaloder Präpositionalphrase) eine verstärkte Konstruktion.

(19a) Der Junge freute sich über die schönen Spielzeuge.

(19b) Der Junge freute sich darüber, daß man ihm so schöne Spielzeuge geschenkt hatte.

Laut Grundannahme (1) behauptet sich die verstärkte Konstruktion vorzugsweise in komplexerem Satzkontext. Auf Textebene ist $\mathrm{zu}$ erwarten, daß die verstärkte Konstruktion eine Affinität zur Grundtextsorte 5 (schriflticher Fernmonolog) zeigt.

Der Großteil aller Obersätze sind Hauptsätze. Daher soll im folgenden vor allem von dem Verhältnis zwischen (selbständigen) Hauptsätzen und abhängigen Sätzen die Rede sein. Hauptsätze sind aufgrund ihrer grundsätzlichen Selbständigkeit prototypische Sätze, Nebensätze aufgrund ihrer grundsätzlichen Unselbständigkeit hingegen nicht. Hauptsätze zeigen allerdings formal komplexere Züge als Nebensätze (Orešnik 1990: 117ff.). Der typische Erscheinungsort von Nebensätzen sind schriftliche Fernmonologe. Distributionsunterschiede zeigen sich auch zwischen mündlichen Dialogen, vermutlich vielfach in Abhängigkeit vom Abstraktionsgrad der Texte. Der Anteil selbständiger Hauptsätze (HS) steigt in weniger abstrakten Texten, während der Anteil von Nebensätzen mit V-Letzt-Stellung (NS) und Infinitivsätzen (IS) in abstrakteren Texten zunimmt. Einen Anhaltspunkt für die Angemessenheit der oben aufgestellten These sind die aus den Textsammlungen IDS2 und IDS3 gewonnenen 
Zahlenangaben in der unten folgenden Tabelle. Unter den dort vertretenen Dialogen (in der Mehrzahl handelt es sich um mündliche Nahdialoge) zeigen die Diskussionen den geringsten Anteil an (selbständigen) Hauptsätzen (etwa 55\%), gefolgt von den Beratungsgesprächen (etwa 65\%), während die Smalltalks und die Dienstleistungsgespräche einen hohen Hauptsatz-Anteil aufweisen (etwa 70\% bzw. mehr als 75\%).

(21) Haupt-, Neben- und Infinitivsatzanteile in Texten des Freiburger Korpus

\begin{tabular}{|l|l|l|l|l|l|l|}
\hline TEXTE & TEXTSORTE & HS\% & ABH. HS\% & NS\% & \multicolumn{1}{|c|}{ IS\% } & SUMME \\
\hline ids3xai & Diskussion & 55.31 & 5.64 & 33.62 & 5.42 & 100.00 \\
\hline ids3xau & Beratungsgespräch & 54.55 & 9.09 & 30.68 & 5.68 & 100.00 \\
\hline ids3xbc & Dienstleistungsgespräch & 77.57 & 3.74 & 17.76 & 0.93 & 100.00 \\
\hline ids3xbh & Dienstleistungsgespräch & 76.19 & 7.14 & 14.29 & 2.38 & 100.00 \\
\hline ids3xcr & Beratungsgespräch & 65.19 & 9.49 & 22.15 & 3.16 & 100.00 \\
\hline ids3xdq & Beratungsgespräch & 65.00 & 11.43 & 20.71 & 2.86 & 100.00 \\
\hline ids3xeg & Smalltalk & 61.71 & 11.34 & 24.18 & 2.77 & 100.00 \\
\hline ids3xel & Beratungsgespräch & 55.83 & 10.00 & 30.83 & 3.33 & 100.00 \\
\hline ids3xeq & Beratungsgespräch & 70.69 & 10.34 & 10.34 & 8.62 & 100.00 \\
\hline ids3xer & Dienstleistungsgespräch & 88.89 & 0.00 & 11.11 & 0.00 & 100.00 \\
\hline ids3xex & Smalltalk & 81.08 & 6.76 & 12.16 & 0.00 & 100.00 \\
\hline ids3xez & Smalltalk & 73.26 & 14.53 & 11.63 & 0.58 & 100.00 \\
\hline ids3xfu & Beratungsgespräch & 76.19 & 4.76 & 16.67 & 2.38 & 100.00 \\
\hline ids3xfv & Smalltalk & 90.00 & 0.00 & 10.00 & 0.00 & 100.00 \\
\hline ids3xhb & Smalltalk & 85.71 & 7.14 & 7.14 & 0.00 & 100.00 \\
\hline ids3yah & Smalltalk & 85.03 & 5.44 & 6.12 & 3.40 & 100.00 \\
\hline ids3ybk & Dienstleistungsgespräch & 83.58 & 1.49 & 11.94 & 2.99 & 100.00 \\
\hline ids3ybl & Smalltalk & 62.50 & 16.67 & 18.75 & 2.08 & 100.00 \\
\hline ids3 & verschiedene & $\mathbf{6 7 . 0 6}$ & $\mathbf{8 . 3 7}$ & $\mathbf{2 1 . 4 6}$ & $\mathbf{3 . 1 2}$ & 100.00 \\
\hline ids2 & Diskussionen & $\mathbf{5 4 . 8 7}$ & $\mathbf{7 . 4 6}$ & $\mathbf{3 2 . 7 8}$ & $\mathbf{4 . 8 8}$ & 100.00 \\
\hline
\end{tabular}

Ein Vergleich zwischen der Textsammlung IDS2 (Heutiges Deutsch 1974), in der öffentliche Diskussionen zu finden sind, und der Textsammlung IDS3 (Heutiges Deutsch 1975), in der verschiedene Textsorten (vor allem Smalltalk, Dienstleistungsund Beratungsgespräche sowie eine öffentliche Diskussion) abgedruckt sind, zeigt, daß die Textsammlung IDS2 einen größeren Anteil von markierteren Konstruktionen aufweist (vgl. die folgende Tabelle), d.h. mehr Nebensätze mit V-Letzt-Stellung (1.), Infinitivsätze (2. ) und Nomina auf -ung (8.). Öffentliche Diskussionen stellen innerhalb der Grundtextsorte mündlicher Nahdialog eine markiertere Textsorte dar. In Texten, die zu den markierteren Grundtextsorten gehören, sind ähnlich hohe Anteile oder sogar höhere Anteile markierterer sprachlicher Konstruktionen zu erwarten. 


\begin{tabular}{|c|c|c|c|c|c|c|}
\hline \multicolumn{7}{|c|}{ (22) Häufigkeit finiter und infiniter Satztypen im Freiburger Korpus } \\
\hline & \multirow[t]{2}{*}{ Vorhersage } & \multicolumn{2}{|c|}{ IDS3 } & \multicolumn{2}{|c|}{ IDS2 } & \multirow[t]{2}{*}{ DI } \\
\hline & & abs. & $\%$ & abs. & $\%$ & \\
\hline \multirow[t]{3}{*}{1.} & Nebensätze (V-Letzt) & 512 & 24.67 & 2405 & 37.92 & -11.00 \\
\hline & Hauptsätze (selbständige) & 1563 & 75.33 & 3938 & 62.08 & \\
\hline & Stichprobengröße (n) & 2075 & 100.00 & 6343 & 100.00 & \\
\hline \multirow[t]{3}{*}{2.} & Infinitivsätze & 73 & 4.46 & 356 & 8.29 & -5.09 \\
\hline & Hauptsätze (selbständige) & 1563 & 95.54 & 3938 & 91.71 & \\
\hline & Stichprobengröße (n) & 1636 & 100.00 & 4294 & 100.00 & \\
\hline \multirow[t]{3}{*}{3.} & Abhängige Hauptsätze & 194 & 11.04 & 544 & 12.14 & -1.21 \\
\hline & Hauptsätze (selbständige) & 1563 & 88.96 & 3938 & 87.86 & \\
\hline & Stichprobengröße (n) & 1757 & 100.00 & 4482 & 100.00 & \\
\hline \multirow[t]{3}{*}{4.} & Eingeschobene Hauptsätze & 9 & 0.57 & 37 & 0.93 & -1.33 \\
\hline & Hauptsätze (selbständige) & 1563 & 99.43 & 3938 & 99.07 & \\
\hline & Stichprobengröße (n) & 1572 & 100.00 & 3975 & 100.00 & \\
\hline \multirow[t]{3}{*}{5.} & Abhängige Sätze & 788 & 33.52 & 3342 & 45.91 & -10.55 \\
\hline & Hauptsätze (selbständige) & 1563 & 66.48 & 3938 & 54.09 & \\
\hline & Stichprobengröße (n) & 2351 & 100.00 & 7280 & 100.00 & \\
\hline \multirow[t]{3}{*}{6.} & Nebensätze (V-Letzt) & 512 & 21.78 & 2405 & 33.04 & -10.33 \\
\hline & restliche Sätze & 1839 & 78.22 & 4875 & 66.96 & \\
\hline & Stichprobengröße (n) & 2351 & 100.00 & 7280 & 100.00 & \\
\hline \multirow[t]{3}{*}{7.} & Infinitivsätze & 73 & 3.11 & 356 & 4.89 & -3.65 \\
\hline & restliche Sätze & 2278 & 96.89 & 6924 & 95.11 & \\
\hline & Stichprobengröße (n) & 2351 & 100.00 & 7280 & 100.00 & \\
\hline \multirow[t]{3}{*}{8.} & Nomina auf -ung(en) & 340 & 1.47 & 1173 & 1.75 & -2.86 \\
\hline & andere Wörter & 22746 & 98.53 & 65710 & 98.25 & \\
\hline & Stichprobengröße (n) & 23086 & 100.00 & 66883 & 100.00 & \\
\hline \multirow[t]{3}{*}{9.} & Nomina auf -ung(en) & 340 & 82.32 & 1173 & 76.72 & 2.44 \\
\hline & infinite Verben in Infinitivsätzen & 73 & 17.68 & 356 & 23.28 & \\
\hline & Stichprobengröße (n) & 413 & 100.00 & 1529 & 100.00 & \\
\hline \multirow[t]{3}{*}{10} & infinite Verben in Infinitivsätzen & 73 & 0.32 & 356 & 0.53 & -4.11 \\
\hline & andere Wörter & 23013 & 99.68 & 66527 & 99.47 & \\
\hline & Stichprobengröße (n) & 23086 & 100.00 & 66883 & 100.00 & \\
\hline
\end{tabular}




\section{ZUSAMMENFASSUNG UND ABSCHLIESSENDE BEMERKUNGEN}

Gemäß der Grundannahme über das Verhalten von sprachlichen Varianten (vgl. Orešnik, Snedec, Teržan, Trobevšek-Drobnak 1991: 5-6) wird zwischen verstärkten Konstruktionen bzw. starken Varianten und geschwächten Konstruktionen bzw. schwachen Varianten unterschieden. Im vorliegenden Aufsatz wurde die Annahme geprüft, ob zwischen verstärkten bzw. geschwächten Konstruktionen einerseits und bestimmten Textsorten andererseits ("Umgebung" einer sprachlichen Konstruktion im weitesten Sinne), eine Affinität besteht. Mit Diewald (1991: 296) haben wir fünf Grundtextsorten unterschieden und diesen Grundtextsorten Markiertheitswerte zugeordnet (siehe (6)). Der mündliche Nahdialog wurde als die am wenigsten markierte Grundtextsorte eingeordnet, der schriftliche Fernmonolog dagegen als die markierteste Grundtextsorte. Die Markiertheitszuordnung geschah aufgrund der Markiertheitsbewertung der situativen Faktoren [dialogisch - monologisch], [mündlich - schriftlich] und [face-to-face - zeitlich und räumlich fern].

Es wurde angenommen, daß der prototypische Erscheinungsort verstärkter Konstruktionen (starker Varianten) in den markierteren Grundtextsorten zu suchen sei, und umgekehrt, daß der prototypische Erscheinungsort geschwächter Konstruktionen (schwacher Varianten) in den weniger markierten Grundtextsorten zu suchen sei. Die folgenden sprachlichen Konstruktionspaare wurden in diesem Aufsatz berücksichtigt: Nominalisierungen auf <-ung> in freien Fügungen vs. Nebensätze, Funktionsverbgefüge vs. einfache Verben, Passiv vs. Aktiv, Futur vs. Präsens pro futuro, Perfekt vs. Präteritum, modalpartikelhaltige Sätze vs. modalpartikellose Sätze, Personalpronomina vs. Nomina, Hauptsätze vs. Nebensätze. Die Affinitäten der verstärkten Konstruktionen zu markierteren Grundtextsorten und die Affinitäten geschwächter Konstruktionen $\mathrm{zu}$ weniger markierten Grundtextsorten können folgendermaßen dargestellt werden:

(23) Markiertheitsskala der Grundtextsorten:

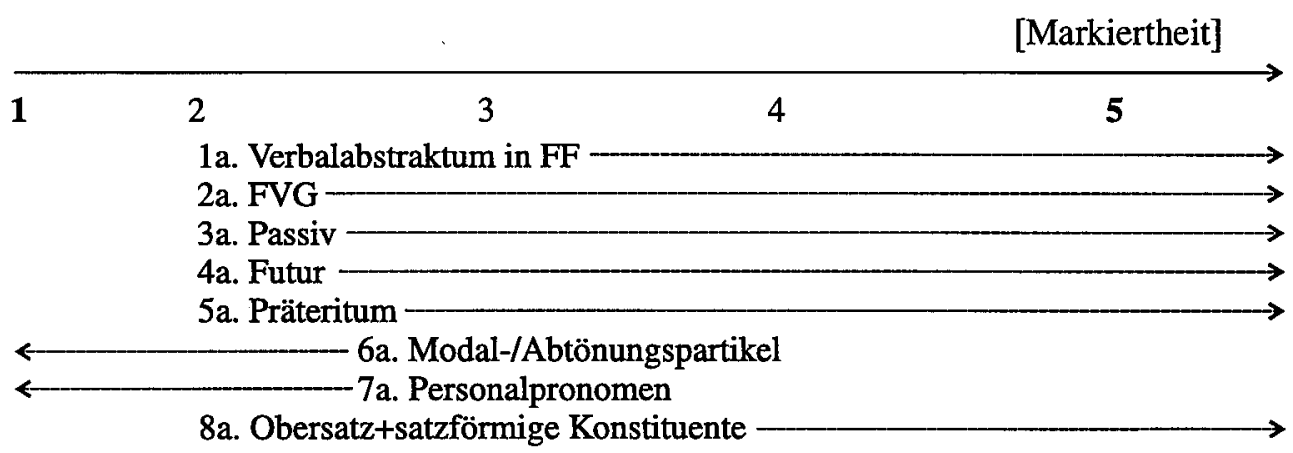




\begin{tabular}{|l|l|c|c|}
\hline \multicolumn{4}{|c|}{ (24) Affinitäten der verstärkten und geschwächten Konstruktionen zu den Grundtextsortenpolen } \\
\hline Konstruktion & Einordnung der Konstruktion & $\mathrm{m}$. N-Dialog & s. F-Monolog \\
\hline 1a. Nominalisierung (in FF) & $\begin{array}{l}\text { geschwächt vs. satzförmige } \\
\text { Konstituente }\end{array}$ & - & + \\
\hline 2a. Funktionsverbgefüge & $\begin{array}{l}\text { verstärkt vs. Satz mit einfachem } \\
\text { Verb }\end{array}$ & - & + \\
\hline 3a. Passiv & verstärkt vs. Aktiv & - & + \\
\hline 4a. Futur & verstärkt vs. Präsens pro futuro & - & + \\
\hline 5a. Perfekt & verstärkt vs. Präteritum & + & - \\
\hline 6a. Satz mit Modalpartikel & $\begin{array}{l}\text { verstärkt vs. modalpartikelloser } \\
\text { Satz (geschwächt vs. Satz + } \\
\text { Kontext + Situation) }\end{array}$ & + & - \\
\hline 7a. Personalpronomen & geschwächt vs. Nomen & + & - \\
\hline $\begin{array}{l}\text { 8a. Obersatz mit satzför- } \\
\text { miger Konstituente }\end{array}$ & $\begin{array}{l}\text { verstärkt vs. Obersatz mit nicht- } \\
\text { satzförmiger Konstituente }\end{array}$ & - & + \\
\hline
\end{tabular}

Zwischen den Kategorien verstärkte Konstruktion (starke Variante) und dem markierteren Grundtextsortenpol 5 (schriftlicher Fernmonolog) sind in folgenden Fällen Affinitäten zu erkennen, die mit der Annahme (4) übereinstimmen: Funktionsverbgefüge (verstärkt) vs. Verb (nicht-verstärkt), Passiv (verstärkt) vs. Aktiv (nicht-verstärkt), Futur (verstärkt) vs. Präsens pro futuro (nicht-verstärkt) und Obersatz mit satzförmiger Konstituente (verstärkt) vs. Obersatz mit nicht-satzförmiger Konstituente (nicht-verstärkt).

Zwischen den Kategorien geschwächte Konstruktion (schwache Variante) und dem weniger markierten Grundtextsortenpol 1 (mündlicher Nahdialog) ist im folgenden Fall eine Affinität zu erkennen, die mit der Annahme (5) übereinstimmt: Personalpronomen (geschwächt) vs. Nomen (nicht-geschwächt).

Nicht-Übereinstimmung mit den Annahmen (4) und (5) liegt in folgenden Fällen vor: Nominalisierung in freier Fügung (geschwächt, aber nicht-prototypische Ausdrucksweise, daher markierter) vs. Nebensatz (nicht-geschwächt), Perfekt (verstärkt, aber prototypischere Ausdrucksweise, daher weniger markiert) vs. Präteritum (nicht-verstärkt), Satz mit Modalpartikel (modal verstärkt, aber prototypische Ausdrucksweise im Deutschen, daher weniger markiert) vs. Satz ohne Modalpartikel (nicht modal verstärkt).

Zusätzliche Faktoren, die die Affinität der Konstruktionen zu bestimmten Textsorten maßgeblich steuern, können angeführt werden (das Pluszeichen bedeutet zutreffende Merkmalsausprägung, das Minuszeichen bedeutet nicht zutreffende Merkmalsausprägung; zu den Faktoren vgl. Diewald 1991):

- Faktoren des Handlungsbereichs: [+privat] [+soziale Nähe] [+symmetrisch] [+freie Themenwahl] (bei Modalpartikeln) 
- Faktoren des Redegegenstandes: [+hohes Abstraktionsniveau] (bei Verbalabstrakta, Passiv, Nebensatz), [+großer Informationsfluß] (bei Verbalabstrakta, Passiv), [+Kontextverschränkung] (bei Futur, Präteritum)

- Faktoren der Textfunktion: [-Kontaktfunktion] (bei Verbalabstrakta, Passiv), [+Darstellung] (bei Präteritum]

Zur Einordnung der Textsorten hinsichtlich ihrer Markiertheit sind neben den situativen Merkmalen auch bestimmte Merkmale anderer Merkmalsachsen (des Handlungsbereiches, der Textfunktion und des Redegegenstandes) relevant, sind aber in der Merkmalhierarchie rangtiefer als die situativen (zur Hierarchie der Merkmalsachsen - Diewald 1991).

Bei der Beurteilung der Markiertheit sprachlicher Varianten ist die Berücksichtigung der Textsortendistribution sprachlicher Varianten insbesondere dann von Nutzen, wenn die Argumente auf anderen Ebenen nicht eindeutig sind:

- Nominalisierungen in freien Fügungen sind hinsichtlich ihrer Textsortendistribution spezialisierter als (bedeutungsäquivalente) Nebensätze. Das ist ein Argument für die Markiertheit der Nominalisierungen.

- Das Präteritum ist hinsichtlich seiner Textsortendistribution eingeschränkter als das Perfekt. Das ist ein Argument für die Markiertheit des Präteritums.

- Funktionsverbgefüge sind dialogferne Formen. Das ist ein Argument für ihre Markiertheit.

- Modalpartikeln zeigen ebenfalls Beschränkungen hinsichtlich ihrer Textsortendistribution. Das wäre ein mögliches Argument für die Markiertheit der Modalpartikeln. Allerdings spricht die Tatsache, daß sie häufig in mündlichen Nahdialogen erscheinen und eine prototypische Ausdrucksweise im Deutschen darstellen, eher für ihre Einfachheit. Daß derartige situative Verweise mit Hilfe von speziellen Lexemen vorgenommen werden, scheint jedoch übereinzelsprachlich weniger typisch zu sein.

- Passiv und Futur sind wiederum typisch für monologische Textsorten. Das ist ein Argument für ihre Markiertheit.

Insgesamt gesehen scheint die Affinität zwischen starken Varianten und markierteren Grundtextsorten einerseits und schwachen Varianten und weniger markierten Grundtextsorten andererseits weniger ausgeprägt zu sein als die Affinität zwischen der Markiertheit von sprachlichen Varianten und markierteren Grundtextsorten einerseits und der Unmarkiertheit von sprachlichen Varianten und weniger markierten Grundtextsorten andererseits. Die Markiertheit von sprachlichen Varianten im Deutschen wurde mit der Fragestellung ermittelt, ob eine bestimmte Ausdrucksweise im Deutschen (d.h. einzelsprachlich) oder übereinzelsprachlich (d.h. auf typologischer oder universeller Ebene) als Default-Ausdruckskategorie für einen bestimmten Inhalt angesehen werden kann. 


\section{LITERATUR}

Boretzky, N. / Dressler, W. / Orešnik, J. / Teržan, K. / Wurzel, W. (1995) (eds.): Natürlichkeitstheorie und Sprachwandel / Teorija naravnosti in jezikovno spreminjanje. Beiträge zum internationalen Symposium über "Natürlichkeitstheorie und Sprachwandel" an der Universität Maribor vom 13.5.-15.5.1993. Bochum.

Brandt, W. (1988): Die Wortlänge in der deutschen Gesetzessprache des 18. bis 20. Jahrhunderts. In: Sprache in Vergangenheit und Gegenwart. Marburg/Lahn. S. 108-121.

Brinker, K. (1971): Das Passiv im heutigen Deutsch. München.

Brons-Albert, R. (1982): Die Bezeichnung von Zukünftigem in der gesprochenen deutschen Standardsprache. Tübingen.

Brons-Albert, R. (1984): Gesprochenes Standarddeutsch. Telefondialoge. Tübingen.

Diewald, G. M. (1991): Deixis und Textsorten im Deutschen. Tuibingen.

Dittmann, J. (1976): Sprechhandlungstheorie und Tempusgrammatik. Futurformen und Zukunftsbezug in der gesprochenen deutschen Standardsprache. München.

Dotter, Franz (1990): Nichtarbitrarität und Ikonizität in der Syntax. Hamburg.

Dressler, W. U. (1989): Semiotische Parameter einer textlinguistischen Natürlichkeitstheorie. Wien.

Gelhaus, H. (1975): Das Futur in ausgewählten Texten der geschriebenen deutschen Sprache der Gegenwart. München.

Grundgesetz (1993): Grundgesetz für die Bundesrepublik Deutschland. Bonn. [GG]

Hentschel, E. (1986): Funktion und Geschichte deutscher Partikeln. Ja, doch, halt und eben. Tübingen.

Hentschel, E. / Weydt, H. (21994): Handbuch der deutschen Grammatik. Berlin, New York.

Heutiges Deutsch (1971): Texte gesprochener deutscher Standardsprache I [= IDS1]. Erarbeitet im Institut für deutsche Sprache, Forschungsstelle Freiburg i. Br. München.

Heutiges Deutsch (1974): Texte gesprochener deutscher Standardsprache II [= IDS2]. Erarbeitet im Institut für deutsche Sprache, Forschungsstelle Freiburg i. Br. München.

Heutiges Deutsch (1975): Texte gesprochener deutscher Standardsprache III [= IDS3]. Erarbeitet im Institut für deutsche Sprache, Forschungsstelle Freiburg i. Br. München.

Krivonosov, A. T. (1977): Deutsche Modalpartikeln im System der unflektierbaren Wortklassen. In: Weydt, H. (1977). S. 176-216.

Latzel, S. (1977): Die deutschen Tempora Perfekt und Präteritum. München.

Leiss, E. (1992): Die Verbalkategorien des Deutschen. Berlin, New York.

Lindner, K. (1983): Sprachliches Handeln bei Vorschulkindern. Tübingen.

Mayerthaler, W. (1981): Morphologische Natürlichkeit. Wiesbaden.

Meier, H. ( $\left.{ }^{2} 1978\right)$ : Deutsche Sprachstatistik. Hildesheim, New York. 
Orešnik (1990): Main vs. Subordinate Clauses: Simple or Complex? In: Dissertationes XIII, Classis II: Philologia et Litterae, Academia Scientarium et Artium Slovenica. Ljubljana. S. 117-127.

Orešnik, J. (1996): Nemški perfekt :: preterit <Deutsches Perfekt :: Präteritum>. Ljubljana. Manuskript.

Orešnik, J. (1997): Ponovitve v luči teorije o krepkih in šibkih zgradbah $<$ Wiederholungen im Lichte der Theorie über die starken und schwachen Konstruktionen>. Linguistični krožek. Ljubljana. Manuskript.

Orešnik, J., Snedec, A., Teržan, K., Trobevšek-Drobnak, F. (1990): Introduction to the Subsequent Three Papers in the Present Volume. In: Linguistica 30. Ljubljana. S. 512

Pavlić, I. (1971): Statistička teorija i primjena. Zagreb.

Petrič, T. (1990): Posamostaljenja v knjižni nemščini <Nominalisierungen im Standarddeutschen $>$. Magisterarbeit. Ljubljana.

Petrič, T. (1993a): Stavki z naklonskimi členki kot okrepljene skladenjske zgradbe <Sätze mit Modalpartikeln als verstärkte syntaktische Konstruktionen>. In: Štrukelj, I. (1993). S. 252-262.

Petrič, T. (1993b): Funktionsverbgefüge und Nominalisierungsverbgefüge im Deutschen als verstärkte syntaktische Konstruktionen im Vergleich zu stammgleichen Verben und Adjektiven. In: Papiere zur Linguistik 49 (1993), 2. S. 125-150.

Petrič, T. (1994): Zu einigen strukturellen Eigenschaften von Nominalisierungen im Deutschen. In: Linguistica 34, 1. "Mélanges Lucien Tesnière". Ljubljana. S. 181-197.

Petrič, T. (1995a): Modalpartikeln und Natürlichkeitstheorie. In: Boretzky, N. / Dressler, W. / Orešnik, J. / Teržan, K. / Wurzel, W. (1995). S. 277-292.

Petrič, T. (1995b): Indexikalische Leistungen der Modalpartikeln und ihre natürlichkeitstheoretische Bewertung. In: Linguistica 35, 1. "Besedilne vrste Textsorten". Ljubljana. S. 245-259.

Petrič, T. (1995c): Nominalisierungen als Beispiel geschwächter syntaktischer Konstruktionen. In: Linguistica 35, 2. Ljubljana. S. 209-256.

Petrič, T. (1995d): Naklonski členki v nemščini <Modalpartikeln im Deutschen>. Dissertation. Ljubljana.

Polenz, P. v. (1987): Funktionsverben, Funktionsverbgefüge und Verwandtes. Vorschläge zur satzsemantischen Lexikographie. In: ZGL 15 (1987), S. 169-189.

Protokoll gesprochene Sprache (1971): Forschungen zur gesprochenen Sprache und Möglichkeiten ihrer Didaktisierung. Protokoll eines Werkstattgesprächs des Goethe-Instituts am 10. und 11. Dezember 1970; veranstaltet vom Referat für Technische Unterrichtsmittel in Zusammenarbeit mit der Forschungsstelle Freiburg des Instituts für Deutsche Sprache Mannheim. München.

Saltveit, L. (1962): Studien zum deutschen Futur. Die Fügung werden mit dem Partizip des Präsens und werden mit dem Infinitiv in ihren heutigen Funktionen und in ihrer geschichtlichen Entwicklung. Bergen, Oslo.

Schwarz, M. (21996): Einführung in die Kognitive Linguistik. Tübingen, Basel. 
So, M.-S. (1991): Die deutschen Funktionsverbgefüge in ihrer Entwicklung vom 17. Jahrhundert bis zur Gegenwart. Eine sprachhistorische Untersuchung anhand von populärwissenschaftlichen Texten. Trier.

Štrukelj, I. (1993) (ed.), Jezik tako in drugače. Ljubljana.

Weydt, H. (1977) (ed.): Aspekte der Modalpartikeln. Studien zur deutschen Abtönung. Tübingen.

Wurzel, W. U. (1984): Flexionsmorphologie und Natürlichkeit. Berlin.

\author{
Povzetek \\ RAZMERJE MED OBLIKOVNO ZAPLETENIMI ALI PREPROSTIMI SKLADENJSKIMI RAZLIČICAMI \\ IN RAZLIČNIMI STOPNJAMI BESEDILNE ZAPLETENOSTI
}

Po osnovni domnevi je možno razlikovati med krepkimi dvojnicami (okrepljenimi zgradbami) in šibkimi dvojnicami (ošibljenimi zgradbami). Krepke dvojnice so zgradbeno in pomensko bolj zapletene od šibkih in se pogosteje pojavljajo $\mathrm{v}$ bolj zapletenem slovničnem okolju, šibke dvojnice pa se pogosteje uveljavljajo $\mathrm{v}$ manj zapletenem. Osrednja tema sestavka je možnost prenosa osnovne domneve s stavčne ravnine na besedilno ravnino. Po Diewaldovi (1991) je avtor sestavka prevzel razvrstitev pet osnovnih besedilnih vrst. Krajevno in časovno neposredni ustni dialog ("mündlicher Nahdialog") je bil ocenjen kot najmanj zaznamovan, krajevno in casovno posredni pisni monolog ("schriftlicher Fernmonolog") pa kot najbolj zaznamovan. Avtorjeva domneva se (v skrajni obliki) glasi: Krepke dvojnice se pogosteje uveljavljajo $\mathrm{v}$ bolj zapletenih (zaznamovanih) osnovnih besedilnih vrstah, šibke dvojnice pa se pogosteje uveljavljajo v manj zapletenih (manj zaznamovanih) osnovnih besedilnih vrstah. Za preverbo domneve avtor primerja naslednje nemške zgradbe: posamostaljenja $z$ odvisniki, funkcijske glagolske zveze s preprostimi glagoli, trpnik s tvornikom, prihodnjik s sedanjikom, dovršilnik s preteritom, stavek + naklonski členek s samim stavkom, osebni zaimek s samostalniško besedno zvezo, nadrejeni stavek s stavčno sestavino nasproti nadrejenemu stavku s samostalniško ali predložno besedno zvezo. V treh primerih zgradbe nimajo afinitete do tistih osnovnih besedilnih vrst, ki bi jo pričakovali glede na zgoraj predstavljeno domnevo: posamostaljenja kot šibke skladenjske zgradbe se v nasprotju $\mathrm{z}$ domnevo pogosteje pojavljajo $\mathrm{v}$ bolj zapletenih besedilnih vrstah, preterit kot šibka glagolska zgradba je v nasprotju z domnevo omejen predvsem na bolj zapletene besedilne vrste, stavki $\mathrm{z}$ naklonskimi členki pa se pogosteje pojavljajo $\mathrm{v}$ manj zapletenih besedilnih vrstah. Na besedilnozvrstno porazdelitev krepkih in šibkih dvojnic vplivajo ne samo situacijski dejavniki, temveč tudi drugi: npr. na porazdelitev naklonskih členkov vplivajo tudi zasebnost, socialna bližina, enakopravnost med udeleženci in prosta izbira teme; na porazdelitev posamostaljenj izdatno vpliva abstraktnost teme in velik obvestilni pretok; na porazdelitev preterita močno vpliva način tematskega razvoja. Avtor sklepa, da je afiniteta krepkih dvojnic do bolj zapletenih (zaznamovanih) besedilnih vrst in afiniteta šibkih dvojnic do manj zapletenih (manj zaznamovanih) besedilnih vrst manj izrazita kot afiniteta zaznamovanih zgradb do zaznamovanih besedilnih vrst in afiniteta nezaznamovanih zgradb do manj zaznamovanih besedilnih vrst. 